\title{
Fungal Biology
}

More information about this series at http://www.springer.com/series/11224 

Monika Schmoll • Christoph Dattenböck Editors

\section{Gene Expression Systems in Fungi: Advancements and Applications}

算 Springer 


\section{Editors}

Monika Schmoll

AIT Austrian Institute of Technology

Tulln, Austria
Christoph Dattenböck

AIT Austrian Institute of Technology

Tulln, Austria

ISSN 2198-7777

ISSN 2198-7785 (electronic)

Fungal Biology

ISBN 978-3-319-27949-7

ISBN 978-3-319-27951-0 (eBook)

DOI 10.1007/978-3-319-27951-0

Library of Congress Control Number: 2016932377

Springer Cham Heidelberg New York Dordrecht London

(C) Springer International Publishing Switzerland 2016

This work is subject to copyright. All rights are reserved by the Publisher, whether the whole or part of the material is concerned, specifically the rights of translation, reprinting, reuse of illustrations, recitation, broadcasting, reproduction on microfilms or in any other physical way, and transmission or information storage and retrieval, electronic adaptation, computer software, or by similar or dissimilar methodology now known or hereafter developed.

The use of general descriptive names, registered names, trademarks, service marks, etc. in this publication does not imply, even in the absence of a specific statement, that such names are exempt from the relevant protective laws and regulations and therefore free for general use.

The publisher, the authors and the editors are safe to assume that the advice and information in this book are believed to be true and accurate at the date of publication. Neither the publisher nor the authors or the editors give a warranty, express or implied, with respect to the material contained herein or for any errors or omissions that may have been made.

Printed on acid-free paper

Springer International Publishing AG Switzerland is part of Springer Science+Business Media (www.springer.com) 


\section{Preface}

Strain improvement with fungal gene expression systems has a tradition of decades regarding modern fermentation industry and even millennia when we think of brewing processes for beer, wine or enzymatic processes in baking. Genetic engineering techniques developed in the last century have revolutionized fermentation industry and enabled production of enzyme mixtures and secondary metabolites with strongly increased efficiency, first by random mutagenesis, but soon also with knowledgebased targeted genome modification. Thereby, economic and optimized production of various compounds originating from diverse organisms became possible in filamentous fungi, from novel, thermostable, or cold active enzymes to potent antibiotics and antibodies to efficient anticancer drugs. At the same time, these possibilities opened up new challenges to be solved, like precise regulation of gene expression during the growth phase for optimized product yield and expression of heterologous proteins with unusual characteristics in a production organism.

This volume aims to give a broad overview on gene expression systems in biotechnological workhorses for production of enzymes and metabolites from the phyla of ascomycetes and basidiomycetes and the subphylum mucoromycotina. Additionally, we include model organisms that have provided valuable insights into the physiology of fungi, also with respect to industrial applications and, for example, the corn smut fungus Ustilago maydis as an alternative expression system. For the diversity in potential fungal hosts for production of a given compound, strain collections and for fungi particularly the Fungal Genetics Stock Center represent an invaluable resource for research and application, which is discussed in a dedicated chapter.

The "Tools" section summarizes current and versatile methods for strain manipulation, such as high throughput construction of genetically modified fungi and strategies for activation of silent secondary metabolite gene clusters and targets for improvement such as the mechanism of carbon catabolite repression. However, also novel strategies and tools that became available in recent years or have raised increased attention are highlighted. The use of inteins in protein synthesis is one example of such intriguing avenues for protein expression, and signal transduction pathways of fungi still remain to be exploited further for optimization of production processes. 
In the last few years also, sexual development was achieved with industrial fungi, which were believed to develop only clonally for decades. Trichoderma reese $i$ was the first one, for which this tool became available and more and more others are following thanks to considerable effort of the fungal scientific community. In this volume we included two chapters on the perspectives and challenges of application of sexual development for strain improvement.

Last but not least, the section on "Challenges" aims to provide insight into the perspectives and needs in industry and discusses safety issues with recombinant production organisms.

With this volume we aim to provide a profound resource for scientists in research and industry alike as well as for students starting their work on application-oriented research with fungi. We intend to inspire researchers to combine classical and novel strategies to meet the challenges with fungal biotechnology in economical and environmentally safe production of the diverse products that make our life more convenient like enzymes or to even save it with novel antibiotics and drugs.

Tulln, Austria

Monika Schmoll Christoph Dattenböck 


\section{Contents}

\section{Part I Organisms}

Gene Expression Systems in Industrial Ascomycetes:

Advancements and Applications

Jonas Ramoni, Verena Seidl-Seiboth, Robert H. Bischof,

and Bernhard Seiboth

Production of Industrial Enzymes in Trichoderma reesei

Marja Paloheimo, Thomas Haarmann, Susanna Mäkinen,

and Jari Vehmaanperä

The Renaissance of Neurospora crassa: How a Classical Model

System is Used for Applied Research

Tanja Seibert, Nils Thieme, and J. Philipp Benz

Improvement of Industrially Relevant Biological Activities in Mucoromycotina Fungi

Tamás Papp, Ildikó Nyilasi, Árpád Csernetics, Gábor Nagy, Miklós Takó, and Csaba Vágvölgyi

Homologous and Heterologous Expression of Basidiomycete

Genes Related to Plant Biomass Degradation.

Sara Casado López, Outi-Maaria Sietiö, Kristiina Hildén,

Ronald P. de Vries, and Miia R. Mäkelä

Prospects for Bioprocess Development Based on Recent Genome

Advances in Lignocellulose Degrading Basidiomycetes .

Chiaki Hori and Daniel Cullen

The Corn Smut Fungus Ustilago maydis as an Alternative

Expression System for Biopharmaceuticals

Parveen Sarkari, Michael Feldbrügge, and Kerstin Schipper 
Gene Expression in Filamentous Fungi: Advantages and Disadvantages Compared to Other Systems.

Nada Kraševec and Mojca Benčina

Yeast Expression Systems for Industrial Biotechnology

Pramote Chumnanpuen, Kanokarn Kocharin, and Wanwipa Vongsangnak

\section{Part II Tools}

High-Throughput Construction of Genetically Modified Fungi

Gyungsoon Park, Shouqiang Ouyang, and Katherine A. Borkovich

Awakening of Fungal Secondary Metabolite Gene Clusters

Juliane Fischer, Volker Schroeckh, and Axel A. Brakhage

Understanding the Mechanism of Carbon Catabolite Repression

to Increase Protein Production in Filamentous Fungi.

Daniel Kiesenhofer, Astrid R. Mach-Aigner, and Robert L. Mach

Inteins and Their Use in Protein Synthesis with Fungi

Skander Elleuche and Stefanie Pöggeler

Relevance of Signal Transduction Pathways for Efficient

Gene Expression in Fungi

Eva Stappler, Aroa Rodriguez-Iglesias, Hoda Bazafkan, Guofen Li, and Monika Schmoll

Sexual Development in Fungi and Its Uses in Gene

Expression Systems

George D. Ashton and Paul S. Dyer

Hybrid Infertility: The Dilemma or Opportunity

of Applying Sexual Development to Improve Trichoderma

reesei Industrial Strains.

Wan-Chen Li, Yu-Chien Chuang, Chia-Ling Chen, and Ting-Fang Wang

Boosting Research and Industry by Providing Extensive

Resources for Fungal Research.

Kevin McCluskey

Systems Biological Applications for Fungal Gene Expression.

Gunseli Bayram Akcapinar and Osman Ugur Sezerman

Part III Challenges in industrial application

Fungal Biotechnology for Industrial Enzyme Production: Focus on (Hemi)cellulase Production Strategies, Advances and Challenges 395

Loreta Gudynaite-Savitch and Theresa C. White 
On the Safety of Filamentous Fungi with Special Emphasis on Trichoderma reesei and Products Made by Recombinant Means

Anna Gryshyna, Liisa Kautto, Robyn Peterson, and Helena Nevalainen

Applications and Benefits of Thermophilic Microorganisms and Their Enzymes for Industrial Biotechnology

Eleni Gomes, Angelica Rodrigues de Souza, Guillermo Ladino Orjuela,

Roberto Da Silva, Tássio Brito de Oliveira, and Andre Rodrigues

Erratum

Index 493

The original version of this book was revised. An erratum to this book can be found at DOI 10.1007/978-3-319-27951-0_22 



\section{Contributors}

Gunseli Bayram Akcapinar, Ph.D. Microbiology Group, Research Area Biotechnology and Microbiology, Institute of Chemical Engineering, Vienna University of Technology, Vienna, Austria

George D. Ashton School of Life Sciences, University of Nottingham, Nottingham, UK

Hoda Bazafkan Department Health and Environment, Bioresources, AIT Austrian Institute of Technology, Tulln, Austria

Mojca Benčina Laboratory of Biotechnology, National Institute of Chemistry, Ljubljana, Slovenia

J. Philipp Benz Holzforschung München, TUM School of Life Sciences Weihenstephan, Technische Universität München, Freising, Germany

Robert H. Bischof Research Division Biotechnology and Microbiology, Institute of Chemical Engineering, Technische Universität Wien, Vienna, Austria

Austrian Centre of Industrial Biotechnology (ACIB) $\mathrm{GmbH}$ c/o Institute of Chemical Engineering, Technische Universität Wien, Vienna, Austria

Katherine A. Borkovich, Ph.D. Department of Plant Pathology and Microbiology, University of California, Riverside, CA, USA

Axel A. Brakhage, Ph.D. Department of Molecular and Applied Microbiology, Leibniz Institute for Natural Product Research and Infection Biology (HKI), Jena, Germany

Chia-Ling Chen Institute of Molecular Biology, Academia Sinica, Taipei, Taiwan Yu-Chien Chuang Institute of Molecular Biology, Academia Sinica, Taipei, Taiwan

Pramote Chumnanpuen Department of Zoology, Faculty of Science, Kasetsart University, Bangkok, Thailand 
Árpád Csernetics, Ph.D. Department of Microbiology, Faculty of Science and Informatics, University of Szeged, Szeged, Hungary

Daniel Cullen, Ph.D. USDA Forest Products Laboratory, Madison, WI, USA

Paul S. Dyer, B.A., M.A., Ph.D. School of Life Sciences, University of Nottingham, Nottingham, UK

Skander Elleuche, Dr. rer. nat. Institute of Technical Microbiology, Hamburg University of Technology (TUHH), Hamburg, Germany

Michael Feldbrügge, Dr. Heinrich Heine University Düsseldorf, Institute for Microbiology, Düsseldorf, Germany

Bioeconomy Science Center (BioSC), c/o Forschungszentrum Jülich, Jülich, Germany

Juliane Fischer, M.Sc. Department of Molecular and Applied Microbiology, Leibniz Institute for Natural Product Research and Infection Biology (HKI), Jena, Germany

Institute of Microbiology, Friedrich Schiller University, Jena, Germany

Eleni Gomes, Ph.D. Laboratory of Biochemistry and Applied Microbiology, Rua Cristóvão Colombo, IBILCE/Unesp, Campus of São Jose do Rio Preto, SP, Brazil

Anna Gryshyna, M.Sc., M.S. Department of Chemistry and Biomolecular Sciences, Macquarie University, Sydney, NSW, Australia

Loreta Gudynaite-Savitch, Ph.D. Iogen Corp., Ottawa, ON, Canada University of Ottawa, Department of Biology, Ottawa, ON, Canada

Thomas Haarmann, Ph.D. AB Enzymes GmbH, Darmstadt, Germany

Kristiina Hildén, Ph.D. Division of Microbiology and Biotechnology, Department of Food and Environmental Sciences, University of Helsinki, Helsinki, Finland

Chiaki Hori, Ph.D. Riken Biomass Engineering Group, Yokohama, Japan

Liisa Kautto, Ph.D. Department of Chemistry and Biomolecular Sciences, Macquarie University, Sydney, NSW, Australia

Daniel Kiesenhofer, Mag. Institute of Chemical Engineering, Vienna University of Technology, Vienna, Austria

Kanokarn Kocharin Bioresources Technology Unit, National Center for Genetic Engineering and Biotechnology (BIOTEC), Pathum Thani, Thailand

Nada Kraševec Laboratory for Molecular Biology and Nanobiotechnology, National Institute of Chemistry, Ljubljana, Slovenia

Guofen Li Department Health and Environment, Bioresources, AIT Austrian Institute of Technology, Tulln, Austria 
Wan-Chen Li Taiwan International Graduate Program in Molecular and Cellular Biology, Academia Sinica, Taipei, Taiwan

Institute of Life Sciences, National Defense Medical Center, Taipei, Taiwan Institute of Molecular Biology, Academia Sinica, Taipei, Taiwan

Sara Casado López, M.Sc. Fungal Physiology, CBS-KNAW Fungal Biodiversity Centre, Fungal Molecular Physiology, Utrecht University, Utrecht, The Netherlands

Robert L. Mach, Ph.D. Institute of Chemical Engineering, Vienna University of Technology, Vienna, Austria

Astrid R. Mach-Aigner, Ph.D. Institute of Chemical Engineering, Vienna University of Technology, Vienna, Austria

Miia R. Mäkelä, Ph.D. Division of Microbiology and Biotechnology, Department of Food and Environmental Sciences, University of Helsinki, Helsinki, Finland

Susanna Mäkinen, M.Sc. Roal Oy, Rajamäki, Finland

Kevin McCluskey, M.S., Ph.D. Department of Plant Pathology, Throckmorton Plant Sciences Center, Kansas State University, Manhattan, KS, USA

Gábor Nagy, Ph.D. Department of Microbiology, Faculty of Science and Informatics, University of Szeged, Szeged, Hungary

Helena Nevalainen, Ph.D. Department of Chemistry and Biomolecular Sciences, Macquarie University, Sydney, NSW, Australia

Ildikó Nyilasi, Ph.D. Department of Microbiology, Faculty of Science and Informatics, University of Szeged, Szeged, Hungary

Tássio Brito de Oliveira, M.Sc. Laboratório de Ecologia e Sistemática de Fungos, IB/Unesp - Campus of Rio Claro, SP, Brazil

Guillermo Ladino Orjuela, M.Sc. Laboratory of Biochemistry and Applied Microbiology, Rua Cristóvão Colombo, IBILCE/Unesp, Campus of São Jose do Rio Preto, SP, Brazil

Shouqiang Ouyang, Ph.D. Department of Plant Pathology and Microbiology, University of California, Riverside, CA, USA

Marja Paloheimo, M.Sc. Roal Oy, Rajamäki, Finland

Tamás Papp, Ph.D. Department of Microbiology, Faculty of Science and Informatics, University of Szeged, Szeged, Hungary

Gyungsoon Park Plasma Bioscience Research Center, Kwangwoon University, Seoul, Republic of Korea

Robyn Peterson, Ph.D. Department of Chemistry and Biomolecular Sciences, Macquarie University, Sydney, NSW, Australia 
Stefanie Pöggeler Department of Genetics of Eukaryotic Microorganisms, Institute of Microbiology and Genetics, Georg-August University-Göttingen, Göttingen, Germany

Jonas Ramoni, M.Sc. Research Division Biotechnology and Microbiology, Institute of Chemical Engineering, Technische Universität Wien, Vienna, Austria

Andre Rodrigues, Ph.D. Laboratório de Ecologia e Sistemática de Fungos, IB/ Unesp - Campus of Rio Claro, SP, Brazil

Aroa Rodriguez-Iglesias Department Health and Environment, Bioresources, AIT Austrian Institute of Technology, Tulln, Austria

Parveen Sarkari, Dr. Institute for Microbiology, Heinrich Heine University Düsseldorf, Düsseldorf, Germany

Kerstin Schipper, Dr. Institute for Microbiology, Heinrich Heine University Düsseldorf, Düsseldorf, Germany

Bioeconomy Science Center (BioSC), c/o Forschungszentrum Jülich, Jülich, Germany

Monika Schmoll, Ph.D. Department Health and Environment, Bioresources, AIT Austrian Institute of Technology, Tulln, Austria

Volker Schroeckh Department of Molecular and Applied Microbiology, Leibniz Institute for Natural Product Research and Infection Biology (HKI), Jena, Germany

Tanja Seibert, M.Sc. Holzforschung München, TUM School of Life Sciences Weihenstephan, Technische Universität München, Freising, Germany

Max Planck Institute of Molecular Plant Physiology, Potsdam, Germany

Bernhard Seiboth Research Division Biotechnology and Microbiology, Institute of Chemical Engineering, Technische Universität Wien, Vienna, Austria

Austrian Centre of Industrial Biotechnology (ACIB) $\mathrm{GmbH}$ c/o Institute of Chemical Engineering, Technische Universität Wien, Vienna, Austria

Verena Seidl-Seiboth Research Division Biotechnology and Microbiology, Institute of Chemical Engineering, Technische Universität Wien, Vienna, Austria

Osman Ugur Sezerman, Ph.D. Department of Biostatistics and Medical Informatics, Acibadem University, Istanbul, Turkey

Outi-Maaria Sietiö, M.Sc. Division of Microbiology and Biotechnology, Department of Food and Environmental Sciences, University of Helsinki, Helsinki, Finland

Roberto Da Silva, Ph.D. Laboratory of Biochemistry and Applied Microbiology, Rua Cristóvão Colombo, IBILCE/Unesp, Campus of São Jose do Rio Preto, SP, Brazil 
Angelica Rodrigues de Souza, Ph.D. Laboratory of Biochemistry and Applied Microbiology, Rua Cristóvão Colombo, IBILCE/Unesp, Campus of São Jose do Rio Preto, SP, Brazil

Eva Stappler Department Health and Environment, Bioresources, AIT Austrian Institute of Technology, Tulln, Austria

Miklós Takó, Ph.D. Faculty of Science and Informatics, Department of Microbiology, University of Szeged, Szeged, Hungary

Nils Thieme, M.Sc. Holzforschung München, TUM School of Life Sciences Weihenstephan, Technische Universität München, Freising, Germany

Csaba Vágvölgyi, D.Sc., Ph.D. Faculty of Science and Informatics, Department of Microbiology, University of Szeged, Szeged, Hungary

Jari Vehmaanperä, Ph.D. Roal Oy, Rajamäki, Finland

Wanwipa Vongsangnak Faculty of Science, Department of Zoology, Kasetsart University, Bangkok, Thailand

Ronald P. de Vries, Ph.D. Fungal Physiology, CBS-KNAW Fungal Biodiversity Centre, Fungal Molecular Physiology, Utrecht University, The Netherland

Ting-Fang Wang, Ph.D. Taiwan International Graduate Program in Molecular and Cellular Biology, Academia Sinica, Taipei, Taiwan

Institute of Molecular Biology, Academia Sinica, Taipei, Taiwan

Theresa C. White, Ph.D. Iogen Corp., Ottawa, ON, Canada

Monsanto Canada Inc., Ottawa, ON, Canada 